\title{
Sobre la ecuación linealizada del vuelo supersónico.
}

\author{
Memoria di Jullo Rey Pastor (a Buenos Aires).
}

Sunto. - Mediante integrales singulares (LEBESGUE-HoBson) se demuestra rigurosamente la conocida solución del caso simétrico en el vuelo supersónico y se aborda el caso antisi. métrico, todavia no resuelto satisfactoriamente. Se determina la zona no perturbada detrais del borde de salida del ala cilindrica finita.

1. Planteo del problema. - Si el movimiento tiene la dirección - $x$, elegido el borde de ataque como eje $y$, o sea si la corriente respecto del ala tiene la dirección $+x$ con velocidad $v_{t}$ respecto del infinito, $\mathrm{y}$ velocidad absoluta $v$, función del punto $P(x, y, z)$ con las hipótesis de irrotacionalidad, y viscosidad nula, existe un potencial $\Phi$ del cual deriva el vector velocidad $\left(\Phi_{x}, \Phi_{y} ; \Phi_{z}\right)$ y que satisface a una ecuación no lineal de $2^{\circ}$ orden. Suponiendo que estos vectores difieren muy poco del vector constante $\left(v_{i}, 0,0\right)$ conviene introducir el potencial de perturbación $\varphi=\Phi-v_{i} x$, cuyas componentes $\varphi_{x}=\Phi_{x}-v_{i}, \varphi_{y}=\Phi_{y}, \varphi_{z}=\Phi_{z}$ deben ser suficientemente pequeñas para poder prescindir de sus produotos y hacer lineal la ecuación. Todavía no se ha estudiado la cuantía del error satisfactoriamente, pero lo cierto es que tales componentes son tanto menores cuanto más pequeña la pendiente del ala (que debe ser sensiblemente plana y muy poco inclinada) y estas condiciones que hacen posible la integración, coinclden afortunadamente con las que impone la realización fisica del vuelo supersónico.

I a ecuación lineal a que satisfacen tanto el potencial $\varphi$ como el $\Phi$ es:

$$
\left(M^{2}-1\right) \varphi_{x x}=\varphi_{y y}+\varphi_{z z} \quad\left(M \text { número de MAOH, función de } v_{i}\right)
$$

y por afinidad, mụltiplicando la coordenada $x$, resulta la ecuación clásica:

$$
L(u)=u_{x x}-u_{y y}-u_{x z}=0 .
$$

Las superficies superior e inferior del ala tienen ecuaciones $z=f^{+}(x, y)$, $z=f^{-}(x, y)$ sobre el recinto $\tau$ planta del ala, con la sola condición de ser muy pequeñas las derivadas $f_{x}, f_{y}$, para la validez de la ecuación lineal, siendo $f^{+} \geq f^{-}$en $\tau$ y $f^{+}=f^{-}$en el contorno.

Las condiciones iniciales impuestas por el problema físico son éstas

a) En el conoide $\Gamma_{0}$ de Mach, envolvente de los conos de semiabertura $\pi / 2$ con vértices en el borde de ataque y dirigidos hacia atrás debo ser $\varphi=0$.

b) En las dos caras del ala debe verificarse por la condición de tangencia: $\varphi_{z}=\left(v_{i}+\varphi_{x}\right), f_{x}+\varphi_{y} f_{y} \approx v_{i} f_{x}$ dentro del segundo orden de aproximación 
y refiriendo cada punto del ala $a$ su proyección sobre el plano $z=0$, las condiciones impuestas sobre las dos caras del recinto plano $\tau$ proyección horizontal del ala son:

$$
\varphi_{a}^{+}\left(x, y, 0^{+}\right)=v_{i} f_{x}^{-}(x, y) \quad \varphi_{z}^{-}\left(x, y, 0^{-}\right)=-v_{i} f_{x}^{-}(x, y) .
$$

La resolución general del problema mixto se reduce a la de dos tipos espèciales: Problema simétrico: (Ala simétrica sin inclinación). Sobre $\tau$ se verifica $f^{+}(x, y)=-f^{-}(x, y), \varphi_{z}^{+}=\varphi_{z}^{-} ;$y por la simetría de las condiciones a) y b) debe ser dentro de $\Gamma_{0}: \varphi(x, y,-z)=\varphi(x, y, z)$. Problema antisimétrico: (Ala sin espesor). Sobre $\tau$ es $f^{-}(x, y)=f^{+}(x, y), \varphi_{z}^{+}=-\varphi_{z}^{-}$y por la antisimetría de las condiciones iniciales dobe ser: $\varphi(x, y,-z)-\varphi(x, y, z)$.

En el caso general, cualesquiera que sean las superficies $f^{+}$e $f^{-}$puede eseribirse :

$$
\begin{array}{ll}
f^{+}=F^{+}+G^{+} & F^{+}=F^{-}=\frac{1}{2}\left(f^{+}+f^{-}\right) \\
f^{-}=F^{-}+G^{-} & G^{+}=-G^{+}=\frac{1}{2}\left(f^{+}-f^{-}\right)
\end{array}
$$

y la solucion del problema general se obtiene sumando las soluciones del problema simétrico $(G)$ y del antisimétrico $(F)$.

Mediante imágenes hidráulicas, acústicas, etc, se ha llegado a la solución del problema simétrico expresada por la integral (2) y el método de VolterRA demuestra que no puede haber otra, pero falta demostrar rigurosamente que esta cumple las condicionas iniciales y satisface a la ecuación; la dada por GUNN mediante método operacional, no es satisfactoria. La teoria de integrales singulares nos permite llenar esta laguna. La solución del problema antisimétrico puede hacerse tambien rigurosamente para alas cilindricas, generalizando así la solución bien conocida del oaso bidimensional (longitud infinita); y así logramos delimitar la zona no pertnrbada para el caso de ala finita, habiéndonos referido por brevedad al caso simétrico. En cuanto a las diversas soluciones que hemos obtenido para el problema general antisimétrico, asi como la dada por el método de VolmerRa y por algún otro ensayo, ninguna es satisfactoria $\mathrm{y}$ todas exigen más profundo análisis. En una memoria extensa que aparecerá en «Collectanea Mathematica» nos ocaparemos de este problema y desarrollaremos las conclusiones resumidas en esta breve nota.

2. Problema simétrico. - Partiendo de la solución elemental $\left(x^{2}-y^{2}-z^{2}\right)^{-\frac{1}{2}}$ correlativa de la $1 / r$ del potencial newtoniano, se forma la familia de soluciones elementales $\left[(x-\xi)^{2}-(y-\eta)^{2}-z^{2}\right]^{-\frac{1}{2}}$ válidas para todo $(\xi, \eta, 0)$ den. tro del cono de vértice $P(x, y, z)$, dirigido hacia adelante, es decir, sobre el recinto $D$ interceptado sobre $\tau$ por ese cono: $(x \quad \xi)^{2} \geq(y-\eta)^{2}+z^{2}$. La inte- 
gral definida sobre ese segmento hiperbólico $D$ :

$$
\varphi(x, y, z)=\iint_{D} \frac{\lambda(\xi ;}{\sqrt{(x-\xi)^{2}-(y-\eta)^{2}-z}}
$$

es convergente, pero no es aplicable la régla de derivación bajo el signo.

Como tampoco es eficaz la regla de DARBoux que considera la integral como parte real de una integrai compleja sobre recinto invariable y tambien presenta difficultades el método de "parte finita» de HADAMARD, nos parece lo más conveniente aplicar el teorema fundamental de las integrales singulares (v. Hobson, vol. II), previo este cambio de variables:

$$
u^{2}=(x-\xi)^{2}-(y-\eta)^{2} \quad v=(x-\xi)+(y-\eta)
$$

que trasforma $D$ en segmento circular $u^{2}+(v-x)^{2} \leq x^{2}, u \geq z$.

La integral del núcleo trasformado se calcula muy elementalmente:

$$
\iint_{D} \frac{d u \cdot \mathrm{d} v}{\sqrt{u^{2}-z^{2}}} \frac{u}{v}=\int_{-\alpha}^{+\alpha} \frac{d w}{w+x} \sqrt{\alpha^{2}-w^{2}} \quad\left(\alpha^{2}=x^{2}-z^{2}\right)
$$

y racionalizada se llega al valor $\pi(x-|z|)$.

Es obvio que el valor de esta integral se puede caleular tambien directamente sin pasar a las variables $u$, $v$, pero así se logra reducir las dos lineas singulares que pasan por el punto $(x, y)$ al solo punto $u=v=0$; y este cambio será útil en otros problemas conexos.

Tanto para la expresiôn (2) del potencial simétrico, come para el antisi. métrico, logramos derivar las expresiones del tipo $\iint_{D} \lambda(u, v) k(u, v, z) d u \cdot d v$ formando el cociente incremental y calculando el limite de la integral análoga, de núcleo $K=\frac{\Delta k}{|z|}$. En el problema simétrico, la integral de este núcleo según el cálculc anterior, vale:

$$
\iint_{D} K(u, v, z) d u \cdot d v=-\pi
$$

y sólo falta ver que la integral sobre todo dominio que excluya el punto singular tiende a 0 para $z \rightarrow 0$. El mismo cálculo realizado para la integración (3) permite evaluar la integral en el intervalo $(h . \alpha)$ siendo fijo el extremo inferior: $|h|<x$. Poniendo $\sqrt{\alpha^{2}-w^{2}}=\alpha-w t$ resulta :

$$
\left[2 x \operatorname{arctg} t-2 z \operatorname{arctg} \frac{x t+\alpha}{z}+\frac{2 \alpha}{1+t^{2}}\right]_{t_{0}}^{1}
$$

con extremo inferior $t_{0}=\frac{\alpha-\sqrt{\alpha^{2}-h^{2}}}{h}=t_{1}+o(z), t_{1}=\frac{x-\sqrt{x^{2}-h^{2}}}{h}$. 
La diferencia de integrales (3) en intervalo $(h, \alpha)$ resulta ser o(z) con evaluaciones muy elementales $y$ por tanto la integral (4) sobre el dominio parcial $D_{1}$ deducido del $D$ por la acotación $v>x-h$, tiende a 0 para $z \rightarrow 0$.

Cumplidas asi las dos condiciones del teorema fundamental de las integrales singulares resulta $\varphi_{z}(x, y, 0)=\pi \lambda(x, y)$. Siendo conocida esta función, per la condición inicial $(b)$, e igual, dentro del $2^{\circ}$ orden de aproximación, a $v_{i} f_{s}(x, y)$ basta sustituir en (2) $\lambda(\xi, \eta)=-\frac{v_{i}}{\pi} f_{\xi}(\xi, \eta)$ para llegar rigurosamente a la clásica fórmula que resuelve en general el problema simetrico.

Conviene observar, que el valor del potencial $\varphi$, y por tanto del vector velocidad, en cada punto interior al conoide $\Gamma_{0}$, no depende de toda el ala, sino solamente de la parte de ella interior al cono anterior de Macr con vértice en aquel punto. Esta circunstancia, especialmente en el caso de ala cilindrica, nos permitirá demostrar la existencia de una zona más o menos extensa detrás del borde de salida, en que el régimen es uniforme, como veremos al final de esta notá.

3. Caso de ala cilíndrica. - La demostración de la fórmula (2) que resuelve el problema simétrico, es inmediata cuando el ala es cilíndrica, y generaliza el resultado bien conocido del caso bidimensional (ala cilindrica infinita) en que por conocerse la integral general de la ecuación de D'ALEMBERT, la solución resulta $\operatorname{ser} \varphi=-v_{i} f(x-|z|)$ en el intervalo $(0, a)$, siendo a la anchura del àla. En efecto, la integración de (2) respecto de $\eta$ es imme. diata, y se llega a:

$$
\varphi(x, y, z)=\iint_{D} \frac{\lambda(\xi, \eta) d \xi \cdot d \eta}{\sqrt{(x-\xi)^{2}-(y-\eta)^{2}-z^{2}}}=\pi \int_{0}^{x-|z|} \lambda(\xi) d \xi
$$

para satisfacer a la condición $(b)$ basta poner $\lambda(\xi)=-\frac{v_{i}}{\pi} f^{\prime}(\xi)$ y se llega a aquella misma solución, mientras que para $x-|z| \geq a$ resulta $\varphi=0$, por ser. $f(0)=f(a)=0$.

Si $P$ está dentro de $\Gamma_{0}$, pero no está dentro del conoide reducido $\Gamma_{0}^{\prime}$, que se deduce suprimiendo los conos cuyos vértices son las puntas $Q^{\prime}$, y $Q^{\prime \prime}{ }_{0}$ del ala subsiste la fórmula (2), pero su integral es más complicada, debiendo distinguirse varios casos. Si el cono de $P$ contiene las puntas $Q_{0}^{\prime}$ y. $Q^{\prime}$, de la derecha, anterior $y$ posterior resulta integrando por partes:

$$
\varphi=\frac{v_{i}(l-\xi)}{\pi} \int_{0}^{a} f(\xi) \frac{x-\xi}{\left[(x-\xi)^{2}-z^{2}\right] \sqrt{(x-\xi)^{2}-(l-\gamma)^{2}-z^{2}}} d \xi
$$

siendo a la anchura y $2 l$ la envergadura del ala. Análogas fórmulas resultan cuando el cono de $P$ contiene las puntas de la izquierda $Q^{\prime \prime}{ }_{0}$ y $Q^{\prime \prime}, \quad$ o bien contiene solamente una punta, o bien tres, o las cuatro. 
Para el problema antisímétrico elegiremos un núcleo impar, resultando satisfactoria la fórmula:

$$
\varphi=\iint_{D} \mu^{\prime}(\xi) \operatorname{arcsen} \frac{z}{\sqrt{(x-\xi)^{2}-(y-\eta)^{2}}} d \xi \cdot d \eta
$$

que integrada respecto de $\eta$ y ealculada después la integral singular que resulta dividiendo por $z$, conduce a este resultado: $\varphi_{z}(x, y, 0)=\pi \mu(0)-\pi \mu(x)$.

Para lograr de acuerdo con la condición $(b)$ que resulte $v_{i} f^{\prime}(x)$ basta adoptar por tanto la expresión:

$$
\varphi=\frac{-v_{i}}{n} \iint_{D} f^{\prime \prime}(\xi) \text { arc sen } \frac{z}{\sqrt{(x-\xi)^{2}-(y-\eta)^{2}}} d \xi \cdot d \eta-(\operatorname{sg} z) v_{i} f^{\prime}(0)(x-|z|)
$$

habiéndose introducido el sumando $x$, para lograr la anulación de $\varphi$ sobre los planos $x= \pm z$,

4. Zona no perturbada. - El hecho, previsto por la integración de la ecuación lineal del problema bidimensional, y comprobade experimentalmente, en alas muy alargadas, de que la perturba ión del campo vectorial uniforme queda confinada a la región situada entro ol conoide de la arista de ataque, formado por dos planos y el engendrado por la arista de salida, formado por otros dos planos paralelos, subsistiendo la uniformidad del campo detrás de este segundo conoide, hace sospechar que en el caso de ala rectangular de envergadura cualquiera $2 l$, la perturbación no llenarà todo el conoide $\Gamma_{0}$ de ataque y quedará una zona más o menos amplia, detrás de la arista de salida en que el régimen seguirá siendo uniforme.

Este problema tridimensional es más complicado que el de ala cilindrica infinita, por no dispouer de la integral general de la ecuación en forma sencilla, como acontecía en aquel problema plano. Sin embargo la solución explicita (2) para el caso simétrico, nos permite llegar a conclusión muy sencilla, delimitando la zona no perturbada, cuya existencia acusan/ los experimentos en túnel, sin precisar su extensión. La comprobación de nuestras conclusiones, aunque sólo sea para el caso de ala cilindrica, afianzaría la validez de la teoria lineal, mientras que un desacuerdo notable obligaría a revisarla, para delimitar su alcance.

Refiriéndonos por brevedad al tipo de ala cilindrica finita, la integración (4) cuando el punto $P$ es interior al conoide de salida $\Gamma_{1}$ y al conoide reducido $\Gamma_{0}^{\prime}$ exige adoptar el intervalo de integración $(0, a)$ y resulta $f(a)-f(0)$, luego el campo de velocidades es uniforme.

Las condiciones analiticas necesarias para la validez de este cálculo son

$$
\text { 1) } \sqrt{x^{2}-z^{2}} \leq l-|y| \quad \text { 2) } x-z \geq a
$$

la primera expresa que los extremos $Q_{0}^{\prime}, Q^{\prime \prime}{ }_{0}$ del borde de ataque no son interiores al cono característico de vértice $P$ y por tanto el intervalo de integra- 
ción en $\eta$ para cada $\xi$ es toda la cuerda interior al cono. Esto se verifica para todo $0 \leq \xi \leq a$, en virtud de la condición 2).

La condición 1) equivale a estas dos:

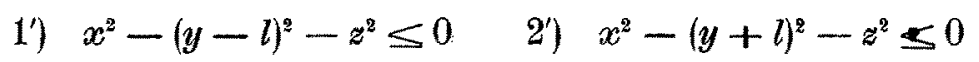

que significan: el punto $P$ no es interior a ninguno de los dos conos de Mach do vértices $Q_{0}^{\prime}$. y $Q^{\prime \prime}{ }_{0}$ puntas del borde de ataque.

Queda asi delimitada la zona de validez del cálculo anterior, en la cual es $\varphi=0$. Suponiendo, como suele acontecer, $l>a$, esta zona no perturbada es una región conexa, simétrica respecto del plano horizontal, en forma de cuña parcial del diedro de MACH engendrado por la recta del borde de salida, con una arista de longitud $2(l-a)$, parte de este borde de salida; y tiene dos puntos cuspidales simétricos rospecto del plano horizonatl, sîtuados en los citados planos de MAcH; puntos cuspidales cuyas coordenadas son:

$$
\left(\frac{b^{2}+a^{2}}{2 a}, 0 ; \pm \frac{b^{2}-a^{2}}{2 a}\right)
$$

Es claro que al pasar de la ecuación simplificada (1) a la ecuación lineal de coeficiente $\left(M^{2}-1\right)$ la abertura de los conos (ángulo de MACH) disminuys al crecer $M$, y la zona no perturbada se prolonga hacia atràs; por otra parte, la anchura de esta zona crece con la envergadura $l$, de modo tal, que para todo $M$, tiende hacia el diedro posterior de Mach, cuyos puntos van quedando todos en esa zona no perturbada, para $l \rightarrow \infty$. 